\title{
Review
}

\section{The role of microsatellite instability in gastric carcinoma}

\section{Background}

During the past four years, the genetic basis of the hereditary non-polyposis colorectal cancer (HNPCC) syndrome has emerged. ${ }^{12}$ Most HNPCC carcinomas exhibit a mutator phenotype characterised by widespread alterations in the length of repetitive DNA sequences (that is, microsatellite instability). A defect in one or more of the known DNA mismatch repair genes results in the disruption of an enzyme system which maintains the integrity of repetitive sequences which are usually stably inherited. ${ }^{3}$ Germline mutations in hMLH1, hMSH2, hPMS1, hPMS2, and possibly GTBP may account for over $90 \%$ of cases of HNPCC. ${ }^{4-8}$

A proportion of HNPCC kindreds (the so-called Lynch II families) are associated with a high frequency of extracolonic carcinomas, most commonly affecting the stomach and endometrium. ${ }^{9}$ Endometrial and gastric carcinomas from HNPCC kindreds show evidence of microsatellite instability. ${ }^{10}$ The relation between microsatellite instability and gastric carcinoma has received considerable attention because of the suggestion that this mutator phenotype may also be found in sporadic carcinomas that are characteristic of the HNPCC syndrome. ${ }^{11}$ The implication is that there may be a group of sporadic gastric carcinomas which are either unrecognised HNPCC cases or a different biological subset of neoplasms, possibly requiring different clinical management.

The literature emerging from both the East and West concerning the role of microsatellite instability in gastric carcinoma is both conflicting and confusing. A number of factors have contributed to this, which include the diversity of populations studied, differences in the quantity and selection of microsatellite loci which are analysed, differences in the definition of a mutator phenotype used by various authors, and studies of small numbers of cases. It is essential to determine whether the detection of microsatellite instability in gastric carcinomas is clinically important or purely of academic interest.

\section{Definitions and methods of detection}

Microsatellites are ubiquitous short repetitive DNA sequences which exhibit length polymorphisms and show variation between individuals. ${ }^{3}$ Microsatellite instability occurs when novel sized alleles are detected in microsatellite sequences derived from carcinoma DNA which are absent in the normal constitutional DNA from the same person. The presence of microsatellite instability at at least one locus may be termed microsatellite instability positive. HNPCC associated carcinomas show microsatellite instability in the majority of microsatellite loci analysed and are categorised as replication error positive (RER+). ${ }^{1}$ There is currently no agreed definition as to the proportion of unstable microsatellite loci required to classify a case as RER+, but it is proposed that such a case should display microsatellite instability in at least $29 \%$ of microsatellite loci examined. ${ }^{1}$ Carcinomas demonstrating instability in less than $29 \%$ of loci may be more appropriately referred to as manifesting low frequency microsatellite instability. The relevance of defining these categories will become apparent.
Polymerase chain reaction (PCR) amplification of microsatellite sequences, followed by electrophoresis of radiolabelled amplification products and autoradiography is the traditional method used to detect microsatellite instability. ${ }^{12}$ Recently, an automated fluorescence PCR microsatellite assay has been successfully developed. This non-radioactive assay facilitates the interpretation of results and enables a rapid throughput of samples. ${ }^{12}$

\section{Prevalence of microsatellite instability and RER in gastric carcinomas \\ SPORADIC CARCINOMAS}

First reports of microsatellite instability in gastric carcinoma emerged from populations with a relatively high incidence of this disease such as the Japanese. Han et al analysed D2S136 and TP53 microsatellite loci with radioactive PCR technology and found that $39 \%$ of cases of sporadic gastric carcinoma were positive for microsatellite instability. ${ }^{13}$ A larger Japanese study found microsatellite instability in $33 \%$ of 76 cases in their analysis of two loci. ${ }^{14}$ This contrasts with sporadic colorectal carcinoma, where frequencies of $9-12 \%$ have been reported. ${ }^{12}{ }^{15}$ Both of the Japanese studies analysed small numbers of microsatellite loci and, as a consequence, reported misleading microsatellite instability frequencies rather than RER+ cases. Their results could have easily been a chance observation.

Nakashima et al examined six markers and reported RER+ in $16 \%$ of their small series of 25 patients. ${ }^{16} \mathrm{~A}$ single Japanese study which used the potentially more accurate fluorescence PCR technique showed that only 5\% of cases had evidence of RER, although overall $23 \%$ showed evidence of microsatellite instability. ${ }^{17}$ It should be emphasised that although these studies examined a larger number of loci, the number of cases analysed was less than 30 . Bearing this in mind, the proportion of Japanese carcinomas which are RER+ may be much lower than suggested by the initial reports which used small numbers of loci.

Because there is considerable geographical variation in the incidence and possibly the aetiology of gastric carcinoma, reports of the prevalence of microsatellite instability in relatively low as well as high risk populations have emerged. One study from the United States has shown that $15 \%$ of carcinomas are RER+. ${ }^{18}$ A German study of 46 gastric carcinomas showed that, despite a frequency of microsatellite instability of $24 \%$, the frequency of RER+ was only $4 \% \cdot{ }^{19}$ Schneider et al used a panel of 42 markers and found that only $5 \%$ of 78 cases were RER $+{ }^{20}$ These data suggest that the frequency of RER+ in gastric carcinomas from Western (relatively low risk) populations is probably similar to that in Japan-that is, up to $15 \%$, despite an overall prevalence of microsatellite instability in the region of $30 \%$. The frequency of RER+ in gastric carcinomas is similar to that observed in sporadic colorectal carcinoma. ${ }^{121521}$

LOW FREOUENCY MICROSATELLITE INSTABILITY VERSUS RER+ Most studies from both the East and West have demonstrated a significant proportion of gastric carcinomas with low frequency microsatellite instability (when $<29 \%$ of loci are affected). This is a frequent observation in 
carcinomas arising in the upper third of the stomach. ${ }^{22}$ In a study of 138 microsatellite loci, Gleeson et al found that only one of 38 gastric cardiac carcinomas was RER+. Almost every remaining case ( $84 \%$ ) showed low frequency microsatellite instability $(0.8-11.4 \%$ of loci examined). The aetiology of this level of instability is as yet undetermined but may reflect a "background" mutation rate in this type of carcinoma or could relate to an intrinsic instability of microsatellite sequences. ${ }^{22}$

\section{Clinicopathological correlations with microsatellite instability and RER}

HISTOPATHOLOGICAL CLASSIFICATION

In colorectal cancer, sporadic cases showing RER+ share clinicopathological features with colorectal carcinomas from HNPCC kindreds. They tend to occur predominantly in the right side of the colon, are mucinous and poorly differentiated. ${ }^{121} 23$ Attempts have been made to correlate the presence of microsatellite instability with a particular clinicopathological profile in gastric carcinoma. However, this is difficult owing to the differences in histopathological classification adopted by Eastern and Western groups, and the heterogeneity in the groups of carcinomas studied.

The Japanese categorise carcinomas according to the degree of tumour differentiation. A statistically significant difference between the incidence of microsatellite instability in poor $(64 \%)$ versus well $(17 \%)$ differentiated carcinomas has been shown. ${ }^{13}$ However, this was contradicted by Semba et al who analysed a larger number of loci and reported a higher frequency in well (33\%) compared with poorly (18\%) differentiated carcinomas (NS). ${ }^{24}$ When carcinomas are classified according to Lauren, European authors have shown that RER+ occurs predominantly in intestinal-type carcinomas but less frequently in the diffuse subtype. ${ }^{19}$

\section{TUMOUR LOCATION AND STAGE}

There is no firm relation between the location of the carcinoma and the presence or absence of RER+. There are reports of microsatellite instability predominantly in the distal stomach ${ }^{16}$ but also fairly equally distributed in sites throughout the stomach. ${ }^{25}$ This is may be related to differences in the populations studied. RER+ has been found in both early and advanced gastric carcinomas ${ }^{26}$ and it is suggested that the frequency increases with advancing stage. ${ }^{1427}$ The detection of microsatellite instability in intestinal metaplasia suggests that replication errors may be present early in the carcinogenic process. ${ }^{24}$ However, the pattern by which RERs accumulate during the progression of gastric carcinoma is as yet undetermined.

\section{Relationship with prognosis}

HNPCC associated colorectal carcinomas are associated with improved survival when compared with sporadic colorectal carcinomas. ${ }^{28} \mathrm{RER}+$ sporadic colorectal neoplasms are also associated with a survival advantage over RER- cases. ${ }^{15} 21$

Dos Santos et al have shown in a study of 61 gastric carcinomas from Portugal, that a greater proportion of the 12 RER+ cases survived to six years compared with the 49 RER- cases $(p<0.05) .{ }^{29}$ Patients with low frequency microsatellite instability had a similar outcome to those without microsatellite instability. The survival difference could be explained by the fact that the frequency of RER+ was $35 \%$ for node negative cases and $14 \%$ among node positive cases $(p<0.05)$. This observation is interesting and requires further exploration on larger numbers of patients.

\section{Association between RER+ and genetic} predisposition

RELATION WITH A POSITIVE FAMILY HISTORY

There are reports of the detection of microsatellite instability in small numbers of patients with a family history of gastric cancer which are interesting but inconclusive. ${ }^{3031}$ In an attempt to establish a genetic link between gastric carcinoma and microsatellite instability, Keller et al found that in cases with a family history of at least two first degree relatives affected by gastric or colon cancer, $56 \%$ were positive for microsatellite instability $(p<0.05) .{ }^{19}$ Of patients with at least two first or second degree relatives affected by stomach or colorectal cancer, $78 \%$ showed microsatellite instability $(\mathrm{p}<0.01)$. Unfortunately, the figures for RER+ in these separate groups were not given. Others have failed to reveal an association between RER+ and any family history of cancer in first degree relatives. ${ }^{16}$ Both of these studies were on small numbers of cases and require confirmation on larger series. Recently, Keller et al used a standardised postal questionnaire to assess family history, ${ }^{32}$ and found that in cases where at least one first degree relative was affected by gastric cancer, $20 \%$ showed RER+. This was higher than the RER+ frequency of $4 \%$ found in randomly selected cases from a previous study of the same population of patients, using similar microsatellite loci. ${ }^{19}$

RELATION WITH MULTIPLE PRIMARY CANCERS

Multiple synchronous gastric cancers are rarer than synchronous colorectal carcinomas. In the colorectum, a higher proportion of carcinomas with microsatellite instability have synchronous neoplasms than those without. ${ }^{12} \mathrm{~A}$ recent study from Japan suggested that $53 \%$ of multiple gastric cancers ${ }^{33}$ showed microsatellite instability compared with $21 \%^{16}$ of solitary primary gastric carcinomas $(p<0.05)$. Although first impressions of these figures are impressive, the frequency of RER+ (at least $29 \%$ of loci demonstrating instability) in the study by Nakashima et al is in fact $30 \%$ among multiple synchronous gastric carcinomas with the remainder showing low level microsatellite instability. Although a statistically significant difference between the frequency of microsatellite instability in these two groups of cases was identified, ${ }^{16}{ }^{33}$ the definitions of RER+ used by Nakashima et al in each study are different and therefore statistical comparisons are inappropriate. An association between RER+ and the development of multiple synchronous gastric carcinomas requires confirmation.

Patients from HNPCC kindreds are at risk of developing a second primary carcinoma and based on this Horii et al have demonstrated an incidence of microsatellite instability in $89 \%$ of 38 patients with multiple primary cancers (including gastric) compared with $11 \%$ in unselected single primary carcinomas $(\mathrm{p}<0.0001) .^{34}$ The prevalence of RER+ was $79 \%$ overall and $60 \%$ among the 25 patients who had a gastric carcinoma. The patients analysed in this study may have a predisposing inherited genetic defect which may or may not be related to HNPCC, and they require further mutational analysis.

The studies of multiple cancers have highlighted some important issues. There is heterogeneity in the detection of microsatellite instability in different carcinomas within the same patient. Of the 30 patients demonstrating RER+ in the study by Horii et al, only 13 (43\%) patients showed this phenomenon in every multiple cancer tested. In Nakashima et al's report, one of the seven RER+ patients showed concordance in the presence of microsatellite instability between carcinomas from the same patient. ${ }^{33}$ These multiple primary carcinomas may either be arising by separate molecular pathways or be different clones which have devel- 
oped on the background of a mutator phenotype. The clinical implication of this heterogeneity is that false negatives could occur if microsatellite instability was being used as a screening tool to detect patients at increased risk of developing cancer.

\section{PREVALENCE OF RER+ IN YOUNG PATIENTS WITH GASTRIC} CANCER

Young patients (under 40 years of age) with gastric carcinoma account for less than $10 \%$ of all cases. They usually have diffuse, poorly differentiated, infiltrating lesions which often arise on the background of histologically "normal" gastric mucosa. It is suggested that these gastric carcinomas are more likely to have a genetic basis than those occurring in older patients, which tend to grow in the presence of chronic atrophic gastritis and intestinal metaplasia. ${ }^{35}$

We have recently investigated a series of 10 young patients with gastric carcinoma from the Yorkshire region using a panel of 11 microsatellite loci and found no evidence of microsatellite instability. ${ }^{36}$ This was somewhat surprising as nine of the 10 cases were poorly differentiated and as many as $64 \%$ of such cases are reported to have microsatellite instability in a Japanese population. ${ }^{13} \mathrm{Al}-$ though our study was based on a small series of patients, the results cast doubt over the role of a mutator phenotype as an important predisposing factor in the development of early onset gastric cancer. This contrasts with the situation in colorectal cancer where $58 \%$ of patients without HNPCC aged under 35 years showed evidence of microsatellite instability, with under a half of those cases being associated with germline DNA mismatch gene mutations. ${ }^{37}$

\section{Mechanism of oncogenesis in RER+ gastric carcinomas}

Until recently, the mechanism by which an increase in the frameshift mutation rate could lead to the development of neoplasia was unknown. Transforming growth factor $\beta$ (TGF- $\beta$ ) is a known inhibitor of cell growth ${ }^{38}$ and exerts its effect by binding to a heterodimer complex containing TGF- $\beta$-RI and TGF- $\beta$-RII receptors. ${ }^{39}$ TGF- $\beta$-RII deactivation renders epithelial cells resistant to the inhibitory effects of TGF- $\beta$, which may lead to uncontrolled growth. ${ }^{40}$ Markowitz et al showed that most colorectal cancer cell lines with RER+ showed deactivation of TGF- $\beta$-RII due to a one or two base deletion affecting a short polyadenine microsatellite sequence, which resulted in a truncated protein. ${ }^{41}$ Recently, a study demonstrated that gastric carcinoma cell lines and five of seven RER+ gastric cancers had frameshift mutations affecting the polyadenine sequence of TGF- $\beta$-RII. ${ }^{42}$ This contrasted with $5 \%$ of RERgastric cancers which showed TGF- $\beta$-RII mutations. This may represent one of possibly many mechanisms by which a mutator phenotype could lead to gastric carcinogenesis.

\section{Mismatch repair gene mutations in RER+ gastric carcinomas \\ SPORADIC GASTRIC CARCINOMAS}

We were unable to identify any reports of mutations in DNA mismatch repair genes in RER+ sporadic gastric carcinomas. Studies of RER+ colorectal carcinomas suggest that mutations of hMLH1 in sporadic cases are rare. ${ }^{43}$ Recently it was reported in a large series of 206 sporadic colorectal carcinomas that $15 \%$ showed evidence of microsatellite instability, and overall only $1 \%$ showed a somatic mutation which occurred in hMLH1 or hMSH2, with no detectable germline mutations identified. ${ }^{44}$ Extrapolating these results would suggest that the frequency of HNPCC associated DNA mismatch repair gene mutations in sporadic RER+ gastric carcinomas would be an extremely rare occurrence. It is possible, however, that inherited or acquired defects in other genes involved in the repair of DNA replication errors may predispose to this mutator phenotype.

PATIENTS WITH A FAMILY HISTORY OF GASTRIC CARCINOMA There are few studies addressing the genetic basis of microsatellite instability in gastric carcinomas. ${ }^{32}$ Keller et al found a novel missense mutation present in hMLH1 from one of 10 patients with an RER+ phenotype who had a positive family history of gastric carcinoma. This mutation would lead to an isoleucine-threonine alteration at codon 655 , but would not lead to a truncated mismatch repair enzyme protein which is commonly observed in HNPCC. ${ }^{5}$ The patient exhibiting an hMLH1 mutation was 71 years old with a 72 year old uncle with a gastric carcinoma. Although this mutation is not typical of HNPCC, it may represent a rare novel DNA mismatch repair gene defect predisposing to the development of gastric carcinoma in this patient. So far, there have been no studies of hMSH2, hPMS1, or hPMS 2 mutations in gastric carcinomas from patients with a positive family history.

\section{Conclusions}

METHODOLOGICAL CONSIDERATIONS

It fundamentally important that authors clearly define the terms used in their analysis of microsatellite instability. The term RER+ should probably be assigned to cases with evidence of microsatellite instability in at least two of seven (or $29 \%$ ) loci. This level of microsatellite instability is suggestive of an HNPCC associated phenotype and may require further analysis. Cases showing instability at smaller numbers of loci should not be discarded, but categorised as low frequency microsatellite instability. Further sequence analysis is required to determine whether or not such cases are linked with mismatch repair gene mutations.

There is currently no consensus as to the optimal number and type of microsatellite markers that should be analysed. The minimum number of loci required to confirm or exclude an RER+ phenotype should be in the range of three to seven markers. ${ }^{110}$ Individual loci are more or less susceptible to the development of microsatellite instability, as shown by a study of gastric carcinoma using 138 markers which revealed an interlocus variability in incidence with a range of 0 to $27 \% .^{22}$ The type of marker analysed may effect the overall prevalence of microsatellite instability. There is currently a great need for this issue to be standardised.

CLINICAL SIGNIFICANCE

The levels of RER+ observed in gastric carcinomas from both Western and Eastern populations is probably in the region of up to $15 \%$. A further proportion of cases demonstrate low frequency microsatellite instability. Based on the literature to date, the analysis of microsatellite instability is currently of limited clinical value outside the context of rare HNPCC cases. Routine analysis of microsatellite instability in sporadic gastric carcinomas would be expensive and yield a small number of RER+ cases, of which only a rare case may turn out to be a new or undiagnosed patient with HNPCC. The detection of RER+ in carcinomas from patients with a strong family history of gastric, colorectal or endometrial cancer may yield some cases which would justify mutational analysis.

FURTHER STUDIES REQUIRED

Whereas the role of microsatellite instability and DNA mismatch repair gene defects in HNPCC is unquestionable and well established, the relevance of this phenomenon in gastric cancer is far from clear. The possibility that 
an RER+ phenotype may predispose to the development of multiple primary cancers needs to be supported by further retrospective and prospective studies. This may lead to the identification of the genetic factors responsible and could highlight a group of patients who would benefit from closer surveillance. The possibility that RER+ status is related to survival requires confirmation on larger numbers of cases. Recently is has been shown that RER+ colorectal carcinoma cell lines are resistant to DNA damage induced by certain cytotoxic agents. ${ }^{45}$ The responsiveness of RER+ gastric carcinomas to chemotherapeutic drugs has yet to be tackled.

Detailed analysis of mutations in hMLH1, hMSH2, hPMS1, hPMS2, and GTBP may elucidate the genetic basis of RER+ gastric carcinomas. So far, no mutational hotspots have been identified in the DNA mismatch repair genes and therefore sequencing for mutations will be a demanding and time-consuming process. The aetiology and importance of low level microsatellite instability is currently unknown and requires further investigation.

\section{Note added in proof}

Wu et al recently investigated hMSH2 mutations in sporadic gastric carcinomas displaying an RER+ phenotype. ${ }^{46}$ They found a somatic GCT to TCT missense mutation at codon 207 (with a predicted alanine to serine amino acid substitution in the hMSH2 protein) in one 41 year old patient with no family history of gastric carcinoma. Although the significance of this abnormality is uncertain, mutations in $\mathrm{hMSH} 2$ may play a role in a small proportion of sporadic gastric carcinomas.

\section{J D HAYDEN \\ I G MARTIN Division of Surgery, University of Leeds, Leeds, UK \\ L CAWKWELL P QUIRKE \\ Division of Molecular Oncology}

Correspondence to: Dr Hayden, Division of Molecular Oncology, Algernon Firth Building, The University of Leeds, Leeds LS2 9JT, UK.

1 Aaltonen LA, Peltomäki P, Leach FS, et al. Clues to the pathogenesis of familial colorectal cancer. Science 1993;260:812-16.

2 Ionov Y, Peinado MA, Malkhosyan S, et al. Ubiquitous somatic mutations in simple repeated sequences reveal a new mechanism for colonic carcinogensis. Nature 1993;363:558-61.

3 Weber JL, May PE. Abundant class of human DNA polymorphisms which can be typed using polymerase chain reaction. Am f Hum Genet 1989;44:388-96.

4 Leach FS, Nicolaides NC, Papodopoulos N, et al. Mutations of a mutS homolog in hereditary nonpolyposis colorectal cancer. Cell 1993;75:121525.

5 Nicolaides NC, Papadopoulos N, Bo L, et al. Mutations of two PMS homologues in hereditary nonpolyposis colon cancer. Nature 1994;371:75-80.

6 Bronner CE, Baker SM, Morrison PT, et al. Mutation in the DNA mismatch repair gene homologue hMLH 1 is associated with hereditary nonpolyposis colon cancer. Nature 1994;368:258-61.

7 Papadopoulos N, Nicolaides NC, Wei Y-F, et al. Mutation of a mutL homolog in hereditary colon cancer. Science 1994;263:1625-9.

8 Papadopoulos N, Nicolaides NC, Liu B, et al. Mutations of GTBP in Papadopoulos N, Nicolaides NC, Liu B, et al. Muta
genetically unstable cells. Science 1995;268:1915-17.

genetically unstable cells. Science 1995;268:1915-17.
9 Lynch HT, Smyrk T, Lynch JF. Overview of natural history, pathology, molecular genetics and management of HNPCC (Lynch syndrome). Int $\mathscr{f}$
manch Cancer 1996;69:38-43.

10 Aaltonen LA, Peltomäki P, Leach FS, et al. Replication errors in benign and malignant tumors from hereditary non-polyposis colorectal cancer patients. Cancer Res 1994;54:1645-8.

11 Peltomaki P, Lothe RA, Aaltonen LA, et al. Microsatellite instability is associated with tumors that characterise the hereditary non-polyposis colorectal cancer syndrome. Cancer Res 1993;53:5853-5.

12 Cawkwell L, Li D, Lewis F, et al. Microsatellite instability in colorectal cancer: improved assessment using fluorescent polymerase chain reaction. Gastroenterology 1995;109:465-71.
13 Han HJ, Yanagisawa A, Kato Y, et al. Genetic instability in pancreatic cancer and poorly differentiated type of gastric cancer. Cancer Res 1993;53:5087-9. 14 Chong J-M, Fukayama M, Hayashi Y, et al. Microsatellite instability in the progression of gastric carcinoma. Cancer Res 1994;54:4595-7.

15 Lothe RA, Peltomäki P, Meling GI, et al. Genomic instability in colorectal cancer: relationship to clinicopathological variables and family history Cancer Res 1993;53:5849-52.

16 Nakashima $\mathrm{H}$, Inoue $\mathrm{H}$, Mori $\mathrm{M}$, et al. Microsatellite instability in Japanese gastric cancer. Cancer 1995;75:1503-7.

17 Toh Y, Oki E, Oda S, et al. An integrated microsatellite length analysis using an automated fluorescent DNA sequencer. Cancer Res 1996;56:2688-91.

18 Strickler JG, Zheng J, Shu Q, et al. p53 Mutations and microsatellite instability in sporadic gastric cancer: when guardians fail. Cancer Res 1994;54:4750-5.

19 Keller G, Rotter M, Vogelsang $\mathrm{H}$, et al. Microsatellite instability in adenocarcinomas of the upper gastrointestinal tract: relation to clinicoadenocarcinomas of the upper gastrointestinal tract: relation to clinico-

20 Schneider B, Pulitzer DR, Brown RD, et al. Allelic imbalance in gastric cancer: an affected site on chromosome arm 3p. Genes Chromosom Cancer $1995 ; 13: 263-71$.

$21 \mathrm{Kim} \mathrm{H}$, Jen J, Vogelstein B, et al. Clinical and pathological characteristics of sporadic colorectal carcinomas with DNA replication errors in microsatellite sequences. Am f Pathol 1994:145:148-56.

22 Gleeson CM, Sloan JM, McGuigan JA, et al. Widespread microsatellite instability occurs infrequently in adenocarcinoma of the gastric cardia. Oncogene 1996;12:1653-62.

23 Thibodeau SN, Bren G, Schaid D. Microsatellite instability in cancer of the proximal colon. Science 1993;260:816-19.

24 Semba S, Yokozaki H, Yamamoto S, et al. Microsatellite instability in precancerous lesion

25 Lin J-T, Wu M-S, Shun C-T, et al. Occurrence of microsatellite instability in gastric carcinoma is associated with enhanced expression of the erbB-2 oncoprotein. Cancer Res 1995a:55:1428-30.

26 Rhyu M-G, Park W-S, Meltzer S. Microsatellite instability occurs frequently in human gastric carcinoma. Oncogene 1994;9:29-32.

27 Lin J-T, Wu M-S, Shun C-T, et al. Microsatellite instability in gastric carcinoma with special references to histopathology and cancer stages. Eur $\mathcal{F}$

28 Sankila R, Aaltonen LA, Jarvinen HJ, et al. Better survival rates in patients with MLH1-associated hereditary colorectal cancer. Gastroenterology 1996; 110:682-7.

29 Dos Santos N, Seruca R, Constancia M, et al. Microsatellite instability at multiple loci in gastric carcinoma: clinicopathological implications and prognosis. Gastroenterology 1996;110:38-44.

30 Zelada-Hedman M, Iselius L, Gunven P, et al. Genetic rearrangements in sporadic and familial gastric carcinomas detected with microsatellite markers. Eur F Surg Oncol 1994;20:667-73.

31 Sasaki A, Nagashima M, Shiseki M, et al. Microsatellite instability in gastric cancer prone families. Cancer Lett 1996;99:169-75.

32 Keller G, Grimm V, Vogelsang H, et al. Analysis for microsatellite instability and mutations of the DNA mismatch repair gene hMLH1 in familial gastric cancer. Int f Cancer 1996;68:571-6.

33 Nakashima $\mathrm{H}$, Inoue $\mathrm{H}$, Honda $\mathrm{M}$, et al. The heterogeneity of microsatellite instability in multiple gastric cancers. Am f Gastroenterol 1995;90:653-6.

34 Horii A, Han H-J, Shimada M, et al. Frequent replication errors at microsatellite loci in tumours of patients with multiple primary cancers. Cancer Res 1994;54:3373-5.

35 Correa P, Shaio Y-H. Phenotypic and genotypic events in gastric carcinogenesis. Cancer Res 1994;54:1941-3S.

36 Hayden JD, Cawkwell L, Sue-Ling H, et al. Assessment of microsatellite alterations in young patients with gastric adenocarcinoma. Cancer 1997;79: 695-8.

37 Liu B, Farrington SM, Petersen GM, et al. Genetic instability occurs in the majority of young patients with colorectal cancer. Nat Med 1995;1:348-52.

38 Moses H, Yang E, Pietenpol J. TGF- $\beta$ stimulation and inhibition of cell proliferation: new mechanistic insights. Cell 1990;63:1005-13.

39 Bassing CH, Howe DJ, Segarini PR, et al. A single heterometric complex is sufficient to mediate biological effects of transforming growth factor- $\beta$ ligand. F Biol Chem 1994;269:14861-4.

40 Park K, Kim S-J, Bang Y-J, et al. Genetic changes in the transforming growth factor $\beta$ (TGF- $\beta$ ) type II receptor gene in human cancer cells: correlation with sensitivity to growth inhibition by TGF- $\beta$. Proc Natl Acad Sci USA 1994;91:8772-6.

41 Markowitz S, Wang J, Myeroff L, et al. Inactivation of the type II TGF- $\beta$ receptor in colon cancer cells with microsatellite instability. Science 1995;268:1336-8.

42 Myeroff L, Parsons R, Kim S-J, et al. A transforming growth factor $\beta$ receptor type II gene mutation common in colon and gastric but rare in endometrial cancers with microsatellite instability. Cancer Res 1996;55: 5545-7.

43 Liu B, Nicolaides NC, Markowitz S, et al. Mismatch repair gene defects in sporadic colorectal cancers with microsatellite instability. Nat Genet 1995; 9:48-55.

44 Konishi M, Kikuchi-Yanoshita R, Tanaka K, et al. Molecular nature of colon tumors in hereditary nonpolyposis colon cancer, familial polyposis, and sporadic colon cancer. Gastroenterology 1996;111:307-17.

45 Branch $\mathrm{P}$, Aquilina G, Bignami M, et al. Defective mismatch binding and a mutator phenotype in cells tolerant to DNA damage. Nature 1993;362 $652-4$.

46 Wu M-S, Sheu J-C, Shun C-T, et al. Infrequent hMSH2 mutations in sporadic gastric carcinoma with microsatellite instability. Cancer Lett 1997;112: $161-6$. 